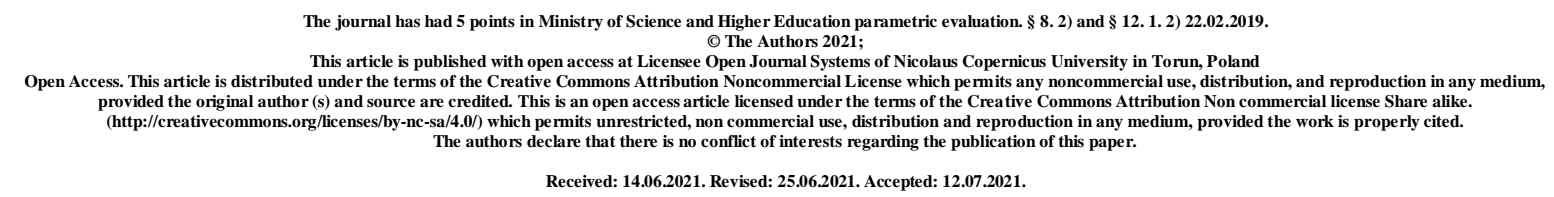

UDK: 611.721.1.018.3.019

\title{
Structural features of the intervertebral disc in rats
}

\author{
M. V. Pankiv, Ye. V. Paltov, Z. Z. Masna, I. V. Chelpanova, M. Ye. Kovalska
}

\section{Danylo Halytsky Lviv National Medical University, Lviv, Ukraine}

M.V. Pankiv, Ph. D-student at the Department of Normal Anatomy, Danylo Halytsky Lviv National Medical University, Lviv, Ukraine; https://orcid.org/0000-0002-3714-2577

Ye.V. Paltov, Ph.D., Associate Professor, Head of the Laboratory of Electron Microscopy, Danylo Halytsky Lviv National Medical University, Lviv, Ukraine; https://orcid.org/0000-0002-2622-4753

Z.Z. Masna, MD, Professor, Head of the Department of Operative Surgery and Topographical Anatomy, Danylo Halytsky Lviv National Medical University, Lviv, Ukraine; https://orcid.org/0000-0003-2057-7061

I.V. Chelpanova, Ph.D., Associate Professor, Department of Histology, Cytology and Embryology, Danylo Halytsky Lviv National Medical University, Lviv, Ukraine https://orcid.org/ 0000-0001-5215-814X

M. Ye. Kovalska Ph.D., Assistant of the department of pathological physiology, Danylo Halytsky Lviv National Medical University, Lviv, Ukraine; https://orcid.org/0000-0002-6232-3951

\section{Abstract}

The aim of our work was to study the structural organization of the components of vertebral disc of rats on the microscopic level in the normal state.

This goal was achieved by means of microscopic method of visualization of cellular components of the intervertebral disc of rats. Histological preparations were made in accordance with the conventional method of using the dye hematoxylin, eosin and azan 
according to the method of Heidenhain, PAS reaction according to MacManus and Alcian blue according to Steedman.

As a result of the microstructural study, it was ascertained that the rat's intervertebral disc normally consists of a gelatinous (pulpal) nucleus and a fibrous ring. It is separated from the vertebral bodies by a thin layer of cartilaginous tissue of the locking plates and hyaline cartilage, which covers the bodies of adjacent vertebrae. The data that rats do not have locking plates can be rarely found in the literature. The vessels verging the intervertebral disc radially -in front, back and from the sides, branch out from the vessels of vertebral periosteum. In the cartilaginous tissue of intervertebral disc, vessels are not visualized.

\section{Key words: intervertebral disc; structural components; norm; rat.}

Results of this article meet the research interests of Danylo Halytsky Lviv National Medical University and are a part of the scientific topic of the Department of Normal Anatomy "Morpho-functional features of organs in pre- and postnatal periods of ontogenesis under condition of opioids and dietary supplements use, reconstructive surgery and obesity" (state registration number 0120U002129) during 2020 - 2024.

Introduction. Intervertebral discs are an essential part of the spine, provide its mobility, and maintain an equal distribution of pressure on the areas of the endplates. This complex structure includes cartilaginous endplates, a fibrous ring and a nucleus pulposus, all of which have no blood supply. Discs receive its nutrition by the vessels of the bone tissue. Therefore, the studies in this field focuse on changes in bone structure. There are some studies which focuse on changes in the mineral density, architecture of vertebral bodies, and the impact of these changes on the direction of degenerative manifestations in the intervertebral discs $[1,2,3]$, the mechanical quality of vertebral bodies and its correlation with the state of intervertebral discs [3]. There are contradictory findings related to the state of the intervertebral disc under conditions of different mineral density. There is a study conducted on twins, which presents a significant connection between disc degeneration and increased mineral density in the proximal femur and lumbar spine [4, 5]. According to other researchers, in the case of low mineral density, intervertebral discs retain their structure $[6,7$, 8]. There is a significant role of subchondral bone tissue in the development of osteoarthritis, which is associated with the production by osteoblasts proinflammatory cytokines and growth factors that affect chondrocytes and close the cycle of arthrosis [9, 10]. If to extrapolate these findings to the bone tissue adjacent to the intervertebral disc, it is assumed that its role is also 
important in the development of destructive disorders in the discs $[11,12,13]$. However, we have not found histological investigations of changes in the intervertebral discs which depend on the condition of the adjacent bone tissue, in particular the bone tissue of the apophyses, although these structures are connected with the endplates by metabolism. [11, 12, 14, 15].

Taking into account all abovementioned, our main goal is to study the features of microstructural organization and nutrition of the intervertebral disc structure in the rats under normal conditions.

Materials and methods. The objects of the study were 15 mature, outbred male rats, weight $80 \mathrm{~g}$, age 4.5 months. All animals were kept in a vivarium, the experiment was conducted in accordance with the principles of bioethics and The European Convention for the Protection of Vertebrate Animals used for Experimental and other Scientific Procedures (Strasbourg, 1986), general ethical principles of animal experiments, approved by the First National Congress of Ukraine on Bioethics (2001), confirmed by the conclusion of members of the Commission on Bioethics of Danylo Halytsky Lviv National Medical University (protocol №10, December 26 ${ }^{\text {th }}, 2011$ ).

Before sampling, the animals were withdrawn from experiment using dibutyl ether. Intervertebral discs of thoracic and lumbar vertebrae of rats with preserved topographic ratio of the structures were used as a material for microstructural investigaton. Histological specimens were prepared according to the typical methods using hematoxylin, eosin, azan and due to Heidenhain method [16], McManus PAS-reaction and Steedman alcian blue method.

Results. As a result of microstructural study, we found that the intervertebral disc, which is located between two adjacent vertebral bodies, consists of a nucleus pulposus and a fibrous ring (Fig. 1).

Intervertebral disc is separated from the vertebral bodies by a thin zone of cartilaginous tissue of the endplates and by a hyaline cartilage, which covers the bodies of adjacent vertebrae. Slightly elongated, elipsoid nucleus pulposus is located in the centre of the intervertebral disc with a slight dorsal displacement, elongated in the dorso-ventral direction and surrounded by collagen fibers. The stroma of the central part of nucleus pulposus is formed by a loose myxoid basic substance (Fig. 2), which barely contains collagen fibers. 


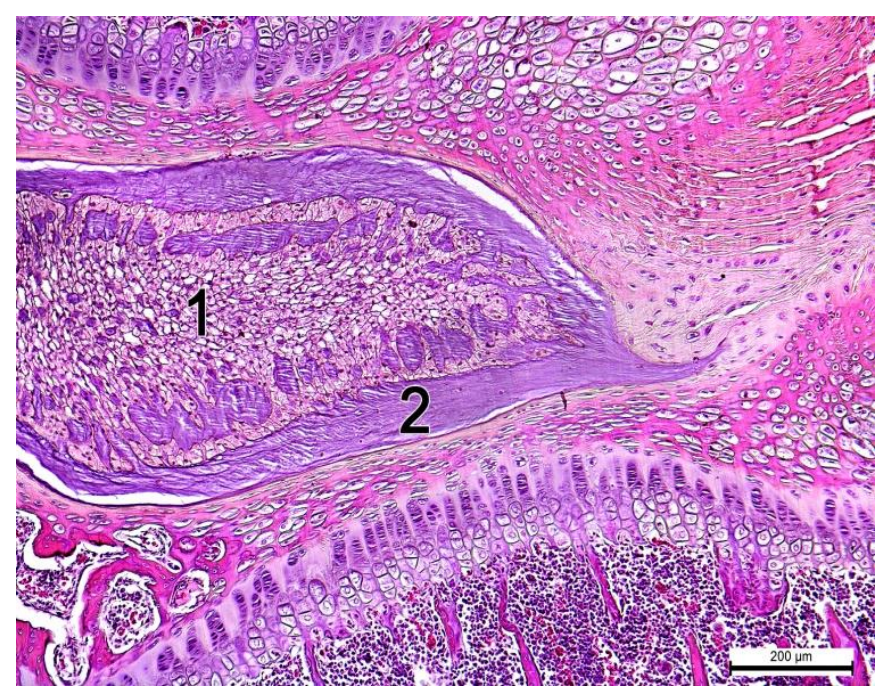

Fig.1. Section of the intervertebral disc of a rat. Staining: hematoxylin and eosin. Photomicrograph x 100 .

1 - nucleus pulposus; 2 - fibrous ring.

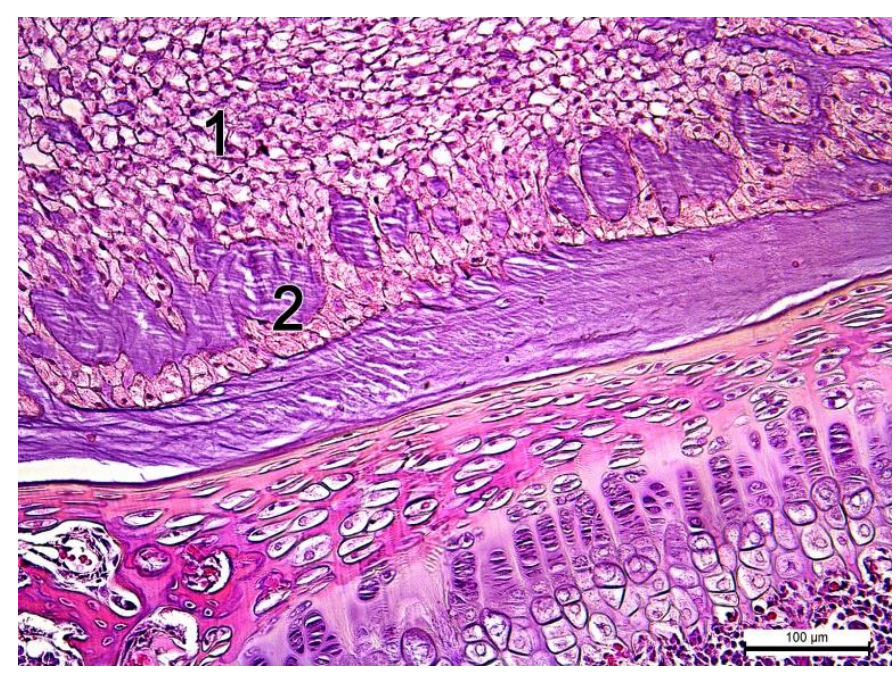

Fig. 2. Section of the intervertebral disc of a rat. Hematoxylin and eosin Photomicrograph. x 200 .

1 - myxoid substance and cellular elements of the nucleus pulposus;

2 - collagen fibers of the fibrous ring which form fibrous patterns.

The intercellular matrix of the outer zone of the fibrous ring contains the identical types of closely packed bundles of collagen fibers (Fig. 3, 4), between which are fibrocytes. The nuclei of fibrocytes are voluminous, rounded with evenly loose heterochromatin, oxyphilic cytoplasm, which forms excrescences. Collagen fibers of the outer zone of the fibrous ring are thicker than in the inner zone, form fibrous patterns, arranged orderly, parallel to the surface of the intervertebral disc and the plane of the vetebra; they intersect with 
adjacent bundles of collagen fibers. The content of primary and acidic glycosaminoglycans in the outer zone of the fibrous ring is slightly higher than in the inner zone. Intervertebral disc proteoglycans are represented by complex macromolecules consisting of proteins and glycosaminoglycans covalently attached to it.

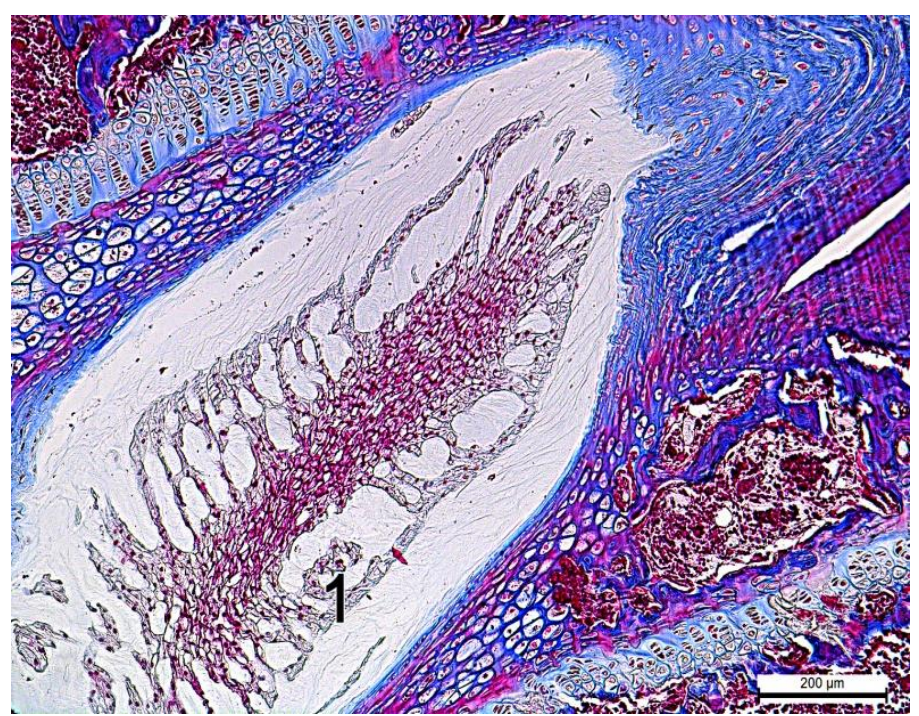

Fig. 3 Section of the intervertebral disc of a rat. Staining: azan due to Heidengain method. Microphotograph x 100.

1 - collagen fibers of the fibrous ring.

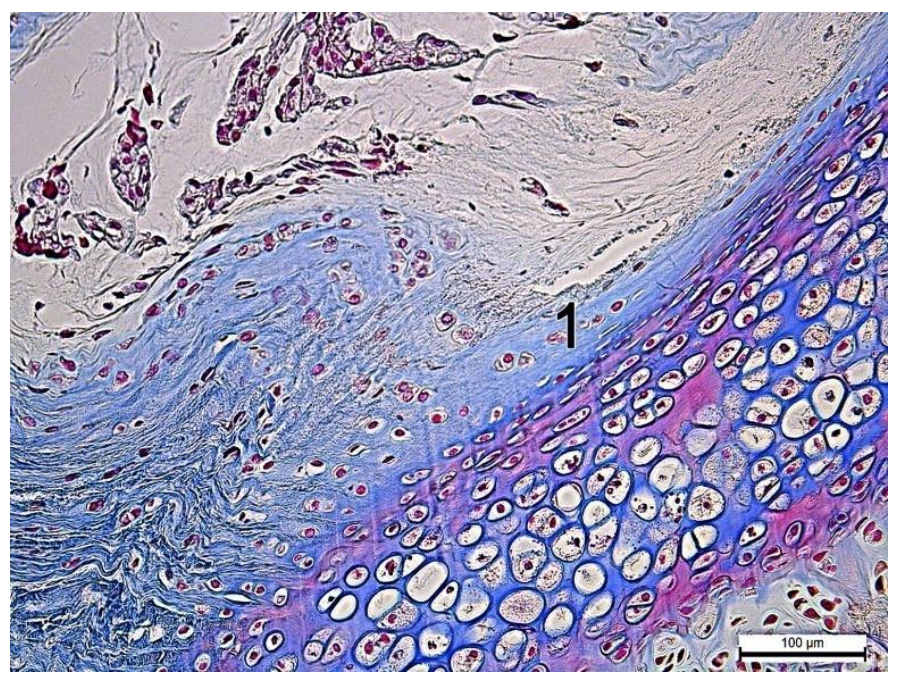

Fig. 4. Section of the intervertebral disc of a rat. Staining: azan due to Heidengain method. Microphotograph x 200 .

1 - collagen fibers of the fibrous ring. 
The main substance of the nucleus pulposus matrix includes a significant amount of primary glycosaminoglycans (Figs. 5, 6), as well as acidic glycosaminoglycans, in the form of voluminous focal rounded patches, metachromatically stained with toluidine blue in the bluegreen color (Fig. 7, 8).

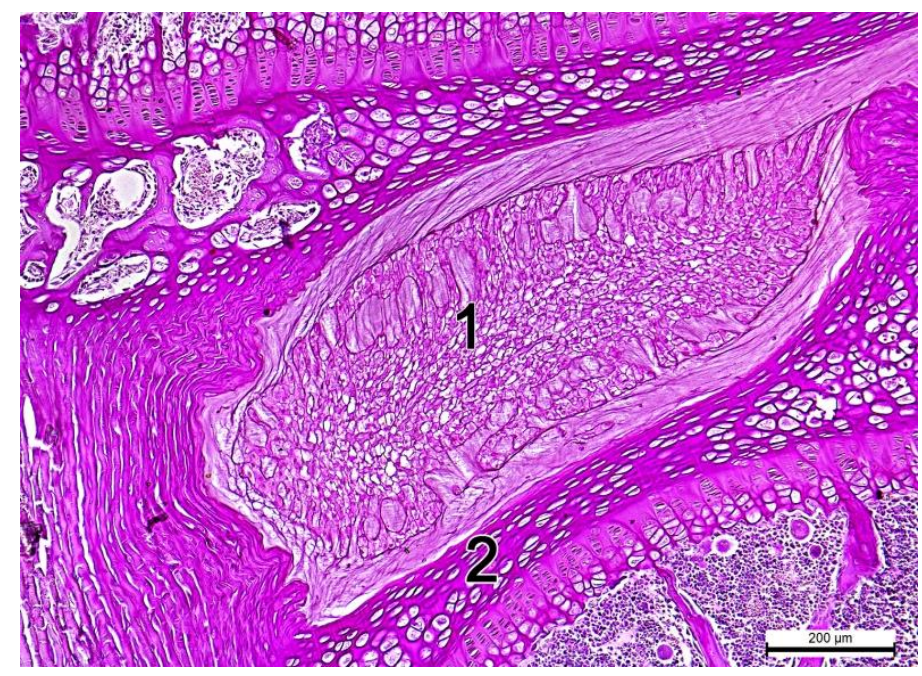

Fig. 5. Section of the intervertebral disc of a rat. Staining: PAS reaction according to McManus method. Microphotograph x 200.

1 - primary glycosaminoglycans of the nucleus pulposus matrix;

2 - primary glycosaminoglycans of the cartilaginous tissue of the intervertebral disc.

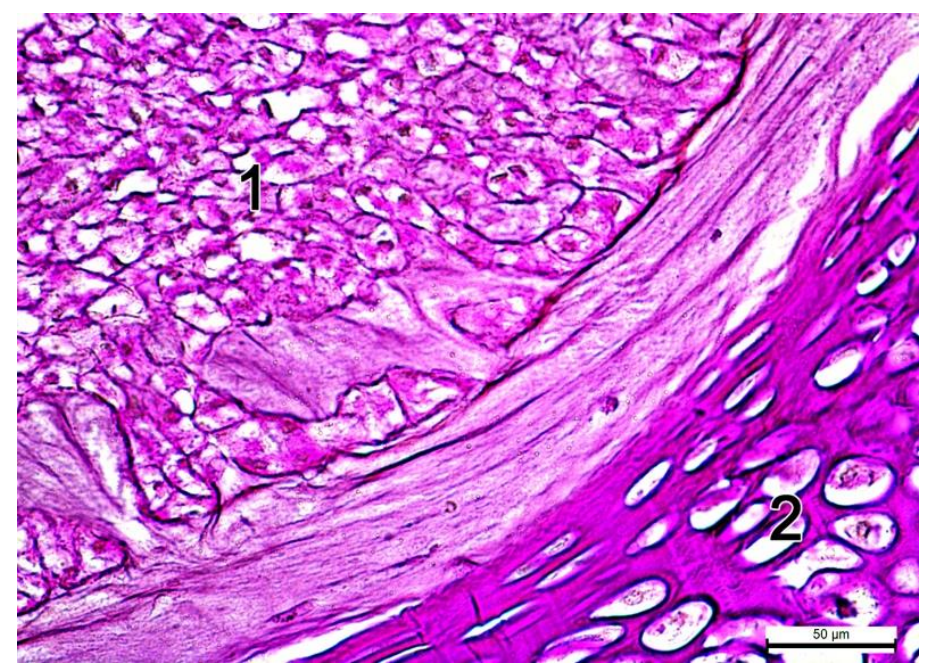

Fig. 6. Section of the intervertebral disc of a rat. Staining: PAS reaction according to McManus method. Microphotograph x 400.

1 - primary glycosaminoglycans of the nucleus pulposus matrix;

2 - primary glycosaminoglycans of the cartilaginous tissue of the intervertebral disc. 
There are two types of cells in the central zone of the nucleus pulposus. Volumetric notochordal cells predominate and contain rounded or oval nuclei, the central parts of which are filled mainly with euchromatin. Their light-colored, moderately vacuolated cytoplasms form excrescences that are connect with the excrescences of adjacent notochordal cells, which result in forming of reticular fibers.

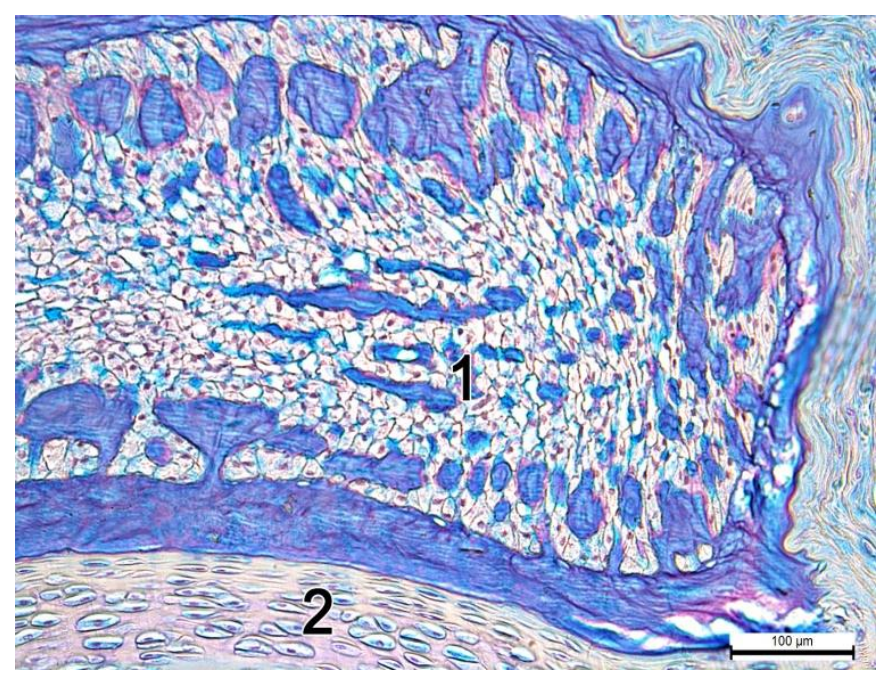

Fig. 7. Section of the intervertebral disc of a rat. Staining: alcian blue according to Steedman method. Photomicrograph. Coll. x 200.

1 - acidic glycosaminoglycans in the nucleus pulposus and fibrous ring;

2 - acidic glycosaminoglycans in the cartilaginous tissue of the intervertebral disc.

Accumulation of notochordal cells form cluster structures somewhere. Less commonly are observed small chondrocyte-like cells with excrescences, which have light euchromatin nuclei and a narrow rim of the cytoplasm in the nucleus pulposus. There are chondroblasts in the peripheral zone of the nucleus pulposus, as well as fibroblasts, which participate in the formation of connective tissue fibers of the nucleus pulposus capsule. Collagen fibers of the nucleus pulposus peripheral zone have a concentric arrangement, some of them are immersed in the stroma of the fibrous ring inner zone.

The tissue of the fibrous ring includes the outer and inner zones. The basis of the fibrous ring inner zone, borders on the nucleus pulposus, is formed by connective tissue, which acquires the features of fibrous cartilage (collagenous fibrous connective tissue). Collagen fibers of the inner zone are thin, their bundles are arranged in the form of collagen (fibrous) lamellae. 


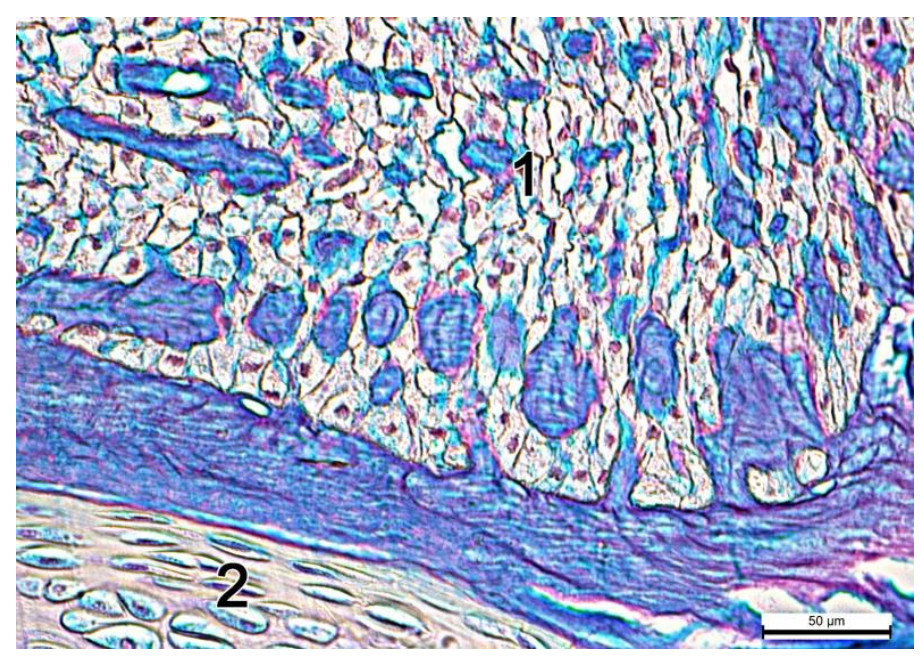

Fig. 8. Section of the intervertebral disc of a rat. Staining: alcian blue according to Steedman method. Microphotograph x 400.

1 - acidic glycosaminoglycans in the nucleus pulposus and fibrous ring;

2 - acidic glycosaminoglycans in the cartilaginous tissue of the intervertebral disc endplate.

The thickness of the collagen fibers of the fibrous ring inner zone is approximately equal; collagen fibers are located around the nucleus pulposus, some of them penetrate into it. Fibrous lamellae are separated by a thin zone of matrix or by sheets of loose connective tissue. The concentration of primary glycosaminoglycans in fibrous lamellae is insignificant. In fibrous lamellae collagen fibers and fibrocyte nuclei are localized in opposite directions, helically relative to the longitudinal axis of the lamella. Collagen fibers of fibrous lamellae are intertwined with each other. After leaving the fibrous lamellae, the collagen fibers intersect with the collagen fibers of adjacent lamellae. Acidic glycosaminoglycans in the collagen fibers of fibrous lamellae are observed more common. Between collagen fibers are visualized the cellular elements of an internal zone of a fibrous ring, which are localized in strictly ordered lacunae, which resemble the chondroblasts. The cells of the inner zone of the fibrous ring are located mostly singly, less often in small, isogenic groups. The intercellular matrix of the inner zone of the fibrous ring in the PAS reaction turns purple, which indicates the presence of a significant amount of primary glycosaminoglycans. Acidic glycosaminoglycans, forming small grains, are localized in large quantities in the cytoplasm of cells of the inner zone of the fibrous ring, as well as in the walls of the lacunae, and, less frequently, in the intercellular substance.

On the periphery of the fibrous ring is the cartilaginous tissue of the endplates, as well as the cartilaginous apophysis of the vertebral body and metaphyseal cartilage. The thin 
endplates consist of cartilaginous tissue, the chondrocytes of which are located in lacunae surrounded by a homogeneous basophilic matrix. The matrix contains a significant amount of primary glycosaminoglycans (Fig. 5, 6), acidic glycosaminoglycans are found somewhere; bundles of collagen fibers were also visualized. Grains of acidic glycosaminoglycans are localized in the cytoplasm of chondrocytes, as well as in the inner wall of the lacunae (Fig. 7, 8).

The cartilaginous elements of the endplates and the metaphyseal cartilage are closely connected with the subchondral areas of the vertebral body and their blood vessels. Elements of the red bone marrow are located in the intertrabecular spaces of the vertebral bodies of the moderate width. From the vessels of the periosteum branch vessels, which approach the intervertebral disc radially - front, back and sides. In the cartilaginous tissue of the intervertebral disc vessels are not visualized.

Future directions. The studies of the structure of the intervertebral discs in the rats will help to form the basis, which can be used for further studies and comparison of opioid effects under normal and pathological conditions.

\section{Conclusions:}

1. The microstructural study showed that the intervertebral disc of the rats normally consists of a nucleus pulposus and a fibrous ring.

2. The intervertebral disc is separated from the vertebral bodies by a thin layer of cartilaginous tissue of the endplates and hyaline cartilage, which covers the bodies of adjacent vertebrae. It is described only few incidences of rats which did not have endplates.

3. From the vessels of the periosteum branch vessels, which approach the intervertebral disc radially - front, back and sides. In the cartilaginous tissue of the intervertebral disc vessels are not visualized.

\section{Conflict of interest: None}

\section{References}

1. Nanjo Y, Morio Y, Nagashima H, Hagino H, Teshima R. Correlation between bone mineral density and intervertebral disk degeneration in pre-and postmenopausal women. J. Bone Miner. Metab. 2003;21(1):22-7. DOI: 10.1007/s007740300004

2. Wang JYX, Kwok AWL, Griffith JF, Leung JCS, Ma HT, Ahuja AT, et al. Relationship Between Hip Bone Mineral Density and Lumbar Disc Degeneration: A Study in 
Elderly Subjects Using an Eight-Level MRI-Based Disc Degeneration Grading System. J Magn Reson Imaging. 2011;33(4):916-20. DOI:10.1002/jmri.22518

3. Wang YX, Griffith JF, Ma HT, Leung JC, Ma HT, Ahuja AT, et al. Is decreased BMD associated with less severe lumbar disc degeneration? Bone. 2008;43(Suppl $1): 35$.

4. Calleja-Agius J, Muscat-Baron Y, Brincat MP. Estrogens and the intervertebral disc. Menopause Int. 2009;15(3):127-30. DOI: 10.1258/ mi.2009.009016

5. Chatterjee R. The effect of low vitamin D on chronic non-specific low back pain: A systematic review Chatterjee R, Hemmings S, Laupheimer MW. International Musculoskeletal Medicine. 2016; 38( 2): 43-50.

6. Rude K, Kirchen ME, Gruber HE, Stashy AA, Meger MH. Magnesiumdeficiency induced bone loss in the rat. Miner, Electrolyte Metal. 1998;24 (5): 314-320.

7. Zhelnin EV, Zvyagintseva TV, Krivoshapka AV. Posttraumatic regeneration of alveolar bone and its relationship with nitric oxide metabolites in glucocorticoid osteoporosis in rats. Successes of modern natural science. 2014; 5 (1): 34-39. [Ukraine]

8. Mattei TA. Osteoporosis delays intervertebral disc degeneration by increasing intradiscal diffusive transport of nutrients through both mechanical and vascular pathophysiological pathways. Med Hypotheses.2013;80(5):582-6. DOI: 10.1016/j.mehy.2013.01.030

9. Povoroznyuk VV, Dedukh NV, Grigorieva NV, Gopkalova IV. K., Experimental osteoporosis. 2012; 228. [Ukraine]

10. Findlay DM, Atkins GJ. Osteoblast-Chondrocyte Interactions in Osteoarthritis Curr. Osteoporos Rep. 2014;12(1):127-34. DOI: 10.1007/ s11914-014-0192-5

11. Keller TS, Ziv I, Moeljanto E. Interdependence of lumbar disc and subdiscal bone properties: a report of the normal and degenerated spine. J Spinal Disord. 1993;6(2):106-13. DOI: 10.1097/00024720-199304000-00003

12. European Convention for the protection of vertebrate animals used for research and other scientific purposes. Strasbourg, March 18, 1986 [Internet] http: zakon.rada.gov.ua/cgi-bin/laws/main.cgi?nreg=994_137

13. Van der Werf M, Lezuo P, Maissen O, van Donkelaar CC, Ito K. Inhibition of vertebral endplate perfusion results in decreased intervertebral disc intranuclear diffusive transport. J. Anat. 2007;211(6):769-774. DOI: 10.1111/j.1469-7580.2007.00816.x 
14. Simpson EK, Parkinson IH, Manthey B, Fazzalari NL. Intervertebral Disc Disorganization Is Related to Trabecular Bone Architecture in the Lumbar Spine. J Bone Miner Res. 2001;(16):681-87. DOI: 10.1359/jbmr.2001.16.4.681

15. Osteoporosis: epidemiology, clinic, diagnosis, prevention and treatment: monograph. under ed. Korzha NA, Povoroznyuk VV, Dedukh NV. Kharkiv: Golden Pages, 2002: 648. [Ukraine]

16. Romeis B. Microscopic technique. M .: Medicine, 1953: 71-72. [Russian] 